\title{
REVISITING BRITTLE FRACTURE AS AN ENERGY MINIMIZATION PROBLEM
}

\author{
G. A. FRANCFORT and J.-J. MARIGO† \\ Institut Galilée, Université Paris-Nord, 93430 Villetaneuse, France
}

\begin{abstract}
A variational model of quasistatic crack evolution is proposed. Although close in spirit to Griffith's theory of brittle fracture, the proposed model however frees itself of the usual constraints of that theory: a preexisting crack and a well-defined crack path. In contrast, crack initiation as well as crack path can be quantified, as demonstrated on explicitly computable examples. Furthermore the model lends itself to numerical implementation in more complex settings.
\end{abstract}

Keywords : fracture, energy release rate, fracture toughness, energy methods, variational calculus.

\section{INTRODUCTION}

Surface energy and energy release rate, the two pillars of brittle fracture theory, stand firm in the landscape of contemporary continuum mechanics. Griffith (1920), their brilliant architect, linked the existence of cracks to surface energy, the macroscopic manifestation of lattice debonding, and the propagation of cracks to the competition between that surface energy and the restitution of bulk energy during an infinitesimal increase of the crack length. Pretty soon, the structure was completed (cf Sih and Liebowitz, 1968) and became a rather formidable citadel.

The fundamental quantities are the toughness (surface energy density), denoted by $G_{c}$ (or $2 \gamma$ ), and by $k$ in the present study, and the energy release rate usually denoted by $G$. Propagation will take place if $G=G_{c}$, and will not if $G<G_{c}$. The literature is of various opinion as to what happens whenever $G>G_{c}$; in any case, the propagation is then labeled unstable, see Nguyen (1987). In the present paper, we will remain focused on one simple task, the capacity for Griffith's theory to predict crack growth. We claim that it is a somewhat unreliable instrument on three grounds:

(i) Crack initiation,

(ii) Crack path,

(iii) Crack jumps along the crack path.

Let us briefly illustrate the above mentioned shortcomings and discuss the merits of available additional tools. 
To illustrate (i) we consider a two-dimensional (2-D) domain filled with a homogeneous, isotropic and linearly elastic material, and containing a straight crack, of length $l$. A mode I excitation of the sample is produced by a load of intensity $t$. For a small crack in a neighborhood of a regular point, the stress intensity factor $K_{\mathrm{I}}$ is of the order of $t \sqrt{l}$ and Irwin's formula yields an energy release rate $G$ of the order of $t^{2} l$. Griffith's criterion will permit propagation for intensities of the order of $1 / \sqrt{l}$. Letting $l$ tend to 0 , we conclude that no crack can appear without an initial crack. Of course, this has to be tempered by the possible presence of singularities in the solution to the crackless elasticity problem; see Subsection 4.4.

The inadequacy of the theory to predict crack path [item (ii) in our three-pronged list] is easily evidenced. In a 2-D setting, the crack tip is parameterized by two functions of time, while Griffith's criterion at best provides one constraint. Additional tests have to be devised so as to determine the proper branching off of the crack, and, to our knowledge, the issue is far from settled, see Amestoy (1987), Leblond (1989) or Amestoy and Leblond (1989) for an insightful analysis of branching in the 2-D, isotropic and homogeneous case. In any case, these analyses bring in extraneous ingredients, which are furthermore limited in scope to very specific classes of cracks, essentially piecewise $\mathscr{C}^{1}$ curves. We will propose a theory that does not a priori constrain the topology of the cracking process, even if some regularity properties of the crack may be obviated a posteriori, although this is, as of yet, a largely open question.

To illustrate (iii), we consider, once again, the example of a 2-D domain filled with a homogeneous, isotropic, and linearly elastic material, and containing a straight initial crack. A mode I excitation of the sample is produced by a load of intensity $t$ and we assume that the crack path will remain straight. If the potential energy is a concave function of the crack length, then Griffith's criterion predicts that the crack will start growing at some time $t_{0}$, but, since $G(t)>k$ when $t>t_{0}$, the criterion does not permit further determination of the crack length after $t_{0}$. The crack is then called unstable, and the analysis is subsequently interrupted. Our formulation however investigates non-smooth crack evolutions and will not stall in such a setting, but will follow up on the crack trajectory after a critical time which may differ from that predicted through Griffith's criterion.

Formulated in and only in a quasistatic setting, the method suggested in Section 2 frees itself of the usual constraints of Griffith's theory, a preexisting crack and a welldefined crack path, but, of course it does so at a price, because it presupposes utter faith in an unsubstantiated postulate, that of global energy minimization for real evolutions. In other words, it is assumed that, as each time, the loaded sample wants to minimize the sum of its bulk and surface energies. Such a postulate is a rather common occurrence in contemporary material science; see e.g. the references at the beginning of Subsection 2.2. But consensus is no guarantee of truth, and the reader should ponder the fairness of such a criterion which further provides no clue as to the ultimate challenge to the citadel, i.e., the dynamic case. On a more personal note, we follow, in our own footsteps, prior work in a similar vein by Francfort and Marigo (1993) or by Fonseca and Francfort (1995). Note also that the seeds of such an approach are present in Ehrlacher and Fedelich (1989).

As will be demonstrated in Subsection 5.2, the proposed formulation can only 
handle, thus far, imposed boundary displacements, but it does so with some degree of generality and efficiency. It applies to any dimension, and any elastic material. It takes into account the whole lifespan of the cracking process from inception to complete failure. It also applies to settings such as delamination, fiber debonding and surface cracks. It is open to non-interpenetration, although the present study is mostly devoted to the no-contact case for the crack lips. The model is presented in Section 2. Section 3 investigates explicit solutions in two model problems. Section 4 examines the general properties of the proposed model; Subsection 4.1 focuses on initiation and failure; Subsection 4.2 compares it to Griffith's theory; Subsection 4.3 is a continuation of that comparison and studies in a detailed manner the brutal vs progressive character of crack growth; Subsection 4.4 revisits the role of singularities in our framework. Section 5 is the problem child: Subsection 5.1 points to possible extensions of the model, while Subsection 5.2 reviews its drawbacks, and suggests hopeful (but not yet operative) remedies. Finally, the short Section 6 glides over the mathematical and numerical questions pertaining to the proposed model; it is not an attempt to offer any kind of mathematical analysis of the proposed model, but rather a brief overview of possible mathematical and numerical venues, together with a partial list of connected open problems; consequently the mathematically inclined reader is urged to consult the references provided there so as to attain a more thorough understanding of the challenges at hand.

\section{A VARIATIONAL FORMULATION OF BRITTLE FRACTURE}

Throughout the paper, $\Omega$ denotes a bounded connected open domain of $\mathbb{R}^{N}$, $1 \leqslant N \leqslant 3$, with smooth boundary $\partial \Omega$ and such that $\Omega$ is the interior of $\bar{\Omega}$. As such $\Omega$ represents the crack-free reference configuration of an elastic body.

In a first subsection the various ingredients of the formulation are introduced; the ensuing evolution problem is described in the second subsection.

\subsection{The ingredients}

Our main partner in this analysis is the crack itself, or rather the family of possible cracked states. In classical fracture mechanics, the family members are very few and pretty much homothetic clones of one another; by contrast, our family is rather extended, since it is made up of all closed subdomains of $\bar{\Omega}$, independently of their shape. Our family has thin members like lines in $2-\mathrm{D}$ or surfaces in $3-\mathrm{D}$; it admits weird members like clouds of points, and marginal members like edge cracks; it is however somewhat prejudiced against fat members, i.e., sets of dimension greater than 1 in 2-D or 2 in 3-D, because those, as will be seen below, do consume too much energy.

An energy is assigned to each member of the family. In the spirit of Griffith, that energy is surface-like and it consists of an average over the crack of the toughness of the material, which is assumed to be a characteristic feature of that material. In other words, if $k(x)$ represents the energy required to create an "infinitesimal" crack at the point $x$ of $\bar{\Omega}$, then the surface energy associated to the crack $\Gamma \subset \bar{\Omega}$ is given by 


$$
E_{s}(\Gamma)=\int_{\Gamma} k(x) \mathrm{d} \mathscr{H}^{N-1}(x),
$$

where $\mathscr{H}^{N-1}$ denotes the $N-1$-D Hausdorff measure (a "surface" measure which amounts to the usual surface measure for smooth enough hypersurfaces, see Evans and Gariepy, 1992).

Remark 2.1. The energy density $k(x)$, the toughness of the given material, is assumed to be strictly positive (and even bounded away from 0), so that energy is created by introducing additional cracks. Furthermore $k(x)$ can take the value $\infty$, which corresponds to a material that would not be breakable at the given point $x$. This will prove useful when analyzing debonding in a composite material.

Remark 2.2. In Subsection 5.1 we will discuss a variant of (1) which departs from Griffith's model; most notably we will introduce a possible dependence of $k$ upon the normal vector to the crack or the jump of the displacement field across the crack; of course, we will then have to be somewhat more restrictive in the criteria that define our family of cracks so as to lend a meaning to objects such as the normal vector.

Remark 2.3. The reader will not have failed to note that closed subsets of $\bar{\Omega}$ with Hausdorff dimension strictly greater than $N-1$ cannot be created unless an infinite amount of energy is spent on their behalf. As will be seen shortly, such a feature prohibits their presence.

We now describe the possible loadings that the sample can undergo. It will become apparent in Subsection 5.2 that the model does not know, as of yet, how to handle forces. This rather unfortunate situation will be elaborated upon in Subsection 5.2; there, we will do our best to minimize the significance of such a restriction upon the validity of our model. Consequently, boundary displacements $U$ on all or part $\partial_{d} \Omega$ of the boundary are the only admissible type of loadings. Specifically, let $\Gamma$ be the actual crack at a given time, the boundary condition is then expressed formally as follows :

(i) on $\partial_{d} \Omega \backslash \Gamma, v=U$, for all kinematically admissible displacement fields, where $U$ is the displacement field which is applied to $\partial_{d} \Omega$,

(ii) on $\partial_{d} \Omega \bigcap \Gamma$ and on $\Gamma \bigcap \Omega$ no displacement is imposed and no boundary conditions are imposed on the kinematically admissible displacement fields,

(iii) on $\partial \Omega \backslash \partial_{d} \Omega$ no displacement is imposed and no boundary conditions are imposed on the kinematically admissible displacement fields.

We should emphasize that we have expressed our boundary conditions as (absence of) restrictions on the kinematically admissible displacement fields rather than as restrictions on the actual displacement field and, correspondingly, on the Cauchy stress (i.e., $\sigma \cdot n=0$ where no forces or displacements are applied). This is so because our formulation will be variational and it is not clear that the ensuing crack possesses enough regularity to lend a meaning to Cauchy stresses.

Remark 2.4. The second item on the above list of possible boundary conditions expresses a no-contact condition on the "lips" of the crack. In the spirit of Remark 2.2 more realistic conditions such as unilateral contact (with or without friction) can 
be imposed at the expense of restricting the class of admissible cracks (cf Subsection 5.1). From now onward, we refer to the adopted setting as the no-contact case.

In conclusion, the kinematically admissible set of displacement fields is a function of both the loading $U$ and the crack $\Gamma$. Its precise definition requires a bit of care because, in the absence of smoothness of $\Omega \backslash \Gamma$, the notion of trace of a displacement field on $\partial(\Omega \backslash \Gamma)$ is debatable. We assume that $\partial_{d} \Omega$ is such that there exists a smooth domain $\Omega_{d}$ containing $\Omega$ such that $\Omega_{d} \cap \partial \Omega=\partial_{d} \Omega$. We also assume that $U$ can be extended to an element $U_{d}$ of $H^{1}\left(\Omega_{d} ; \mathbb{R}^{N}\right)$ - the usual Sobolev space of functions of $L^{2}\left(\Omega_{d} ; \mathbb{R}^{N}\right)$ with weak derivatives in $L^{2}\left(\Omega_{d} ; \mathbb{R}^{N}\right)$-so that $U_{d \mid \partial_{d} \Omega}=U$. We then define the set of kinematically admissible displacement fields as

$$
\mathscr{C}(\Gamma, U)=\left\{v \in H^{1}\left(\Omega_{d} \backslash \Gamma ; \mathbb{R}^{N}\right) \mid v=U_{d} \text { in } \Omega_{d} \backslash \bar{\Omega}\right\} .
$$

Remark 2.5. The above definition implicitly forces the admissible displacements to take the value $U$ on $\partial_{d} \Omega \backslash \Gamma$, provided that such a trace makes sense.

The third ingredient on our agenda is material behavior. In all that follows the material is assumed to behave elastically and to only undergo infinitesimal transformations. Note that the extension to finite elasticity is easily performed in the variational framework proposed below; we will not dwell any further on finite transformations but refer the interested reader to Fonseca and Francfort (1995) for a mathematical study of a (hopefully) closely related model within that framework. The extension to inelastic behavior however should be considered well beyond the scope of the model.

If $\varepsilon(v)$ denotes the symmetrized gradient of $v$, an elastic energy density $W(x, \varepsilon(v)(x))$ is thus given at each point $x$ of $\Omega \backslash \Gamma$. We further assume the following properties for $W$ :

(i) $W(x, \xi)$ is a Carathéodory function-i.e., measurable in $x$ and continuous in $\xi$ for almost all $x$-strictly convex and homogeneous of degree $p, p>1$, in $\xi$,

(ii) $\left.\alpha|\xi|^{p} \leqslant W(x, \xi) \leqslant \beta|\xi|^{p}+1\right), \alpha, \beta>0, \xi$ symmetric.

In fact we will mostly devote our attention to linearized elasticity whereupon (i), (ii) are replaced by

$$
W(x, \xi)=\frac{1}{2} A(x) \xi \cdot \xi,
$$

with

$$
A(x) \text { symmetric and s.t. } \alpha I \leqslant A(x) \leqslant \beta I \text { for a.e. } x \in \Omega .
$$

Note however that all obtained results are immediately generalizable to the nonlinear (but convex and $p$-homogeneous) setting.

We accordingly define the bulk energy as

$$
E_{d}(\Gamma, U)=\inf _{v \in \mathscr{G}(\Gamma, U)} \int_{\Omega_{\Gamma}} W(x, \varepsilon(v)(x)) \mathrm{d} x .
$$

Remark 2.6. Note that the bulk energy is defined as an infimum, and not a minimum; in the absence of regularity properties for $\partial \Omega \backslash \Gamma$, it is not clear that the infimum is 
attained because Korn's inequality may not hold on such a domain. Of course, when the elastic equilibrium of $\Omega_{\Gamma}=\Omega \backslash \Gamma$ is achieved by a displacement field $u \in \mathscr{C}(\Gamma, U)$, then the bulk energy is $\int_{\Omega_{\Gamma}} W(x, \varepsilon(u)(x)) \mathrm{d} x$.

Note also that the independence of $E_{d}(\Gamma, U)$ upon the choice of the extended domain $\Omega_{d}$ remains to be established.

Remark 2.7. The energies $E_{d}$ and $E_{s}$ satisfy the following elementary properties:

(i) $E_{s}$ is strictly monotonically increasing in $\Gamma$,

(ii) $E_{d}$ is monotonically decreasing in $\Gamma$ for any fixed $U$,

(iii) whenever (3) is satisfied, $E_{d}$ is positively homogeneous of degree 2 in $U$.

The total energy of the body for a given crack $\Gamma$ and a given loading $U$ is then given by

$$
E(\Gamma, U)=E_{d}(\Gamma, U)+E_{s}(\Gamma) .
$$

\subsection{The evolution law}

We now propose to follow the response of the body to a time-dependent loading. To this effect a time-parameterized loading $U(t)$ is applied to $\partial_{d} \Omega$. Assume that a (maybe empty) initial crack $\Gamma_{0}$ is present in the body at the onset of the loading process. Our goal is to determine the evolution of the crack(s) during the loading, i.e., to obtain the time-parameterized mapping $\Gamma(t)$. The basic idea is as follows. At a given time $t$, and for the corresponding loading $U(t)$ the crack $\Gamma(t)$ will be the closed subset of $\bar{\Omega}$ which minimizes $E(\Gamma, U(t))$ among all cracks $\Gamma$ which contain all previous $\Gamma(s), s<t$.

There are two important features in this formulation. On the one hand, the driving principle is global energy minimization. Such a principle is not dictated by any known thermodynamical argument; it is rather a convenient postulate which provides for useful insight into a variety of behaviors ranging from martensitic-austenitic phase transitions, see Ball and James (1987), to damage, see Francfort and Marigo (1993), or magnetostriction, see James and Kinderlehrer (1993)... . A more realistic approach that would investigate local minimizers is doomed for want of the necessary mathematical apparatus. On the other hand, the geometry and size of the crack is limited by its predecessors; this is an attempt at expressing the irreversibility of the cracking process, and the absence of healing.

If the conceptual principle is fairly clear, its precise formulation is not so easily laid out. We proceed in two steps, the first of which addresses time-discretized evolutions, while the second pertains to time-continuous evolutions, albeit for a specific subclass of all possible loadings.

\section{Law 2.8 Discrete evolutions:}

Assume that $\Gamma_{0}$ is given. Let $U_{i}, 1 \leqslant i \leqslant p$ be a sequence of loadings. Then, the corresponding cracks $\Gamma_{i}$ have to satisfy

$$
\Gamma_{i} \supset \Gamma_{i-1}, \quad E\left(\Gamma_{i}, U_{i}\right) \leqslant E\left(\Gamma, U_{i}\right) \quad \text { for every } \Gamma \supset \Gamma_{i-1} .
$$

As such, the evolution is discretization-dependent. The real evolution should be 
construed as a limit of the discrete evolution as the time-step tends to 0 . It is not so clear to us that a precise limit can then be derived for all loading scenarios and we will not venture down such a path, but rather will investigate a subclass of loadings for which the limit process is (at least formally) sound, that of monotonically increasing loadings henceforth labeled "MIL". Assume therefore that the loading is of the form

$$
U(x, t)= \begin{cases}t U_{0}(x), & t \geqslant 0, \\ 0 & t<0,\end{cases}
$$

for all $x$ 's on $\partial_{d} \Omega$.

There is nothing remarkable about the linear dependence of $U$ upon $t$, and any increasing function $f(t)$ of $t$ would equally do.

\section{Law 2.9 Continuous monotone evolutions :}

Let $U(t)$ be a MIL. Then, the corresponding cracks $\Gamma(t)$ have to satisfy

(i) $\Gamma(t) \nearrow$ with $t$, and $\Gamma(t)=\Gamma_{0}, t<0$,

(ii) $E(\Gamma(t), U(t)) \leqslant E(\Gamma, U(t))$, for all $\Gamma \supset \bigcup_{s<t} \Gamma(s)$,

(iii) $E(\Gamma(t), U(t)) \leqslant E(\Gamma(s), U(t))$, for all $s<t$.

The above Law immediately calls for a few comments. The first condition is merely the requirement that the crack increases with time from its original state $\Gamma_{0}$. We will actually show in Remark 2.10 below that $\Gamma(0)=\Gamma_{0}$. That the energy should be minimum among all possible cracks at a given time is expressed in the second condition which can be formally derived from Law 2.8 upon replacing $t_{i}$ by $t$ and $\Gamma_{i-1}$ by $\bigcup_{s<t} \Gamma(s)$. The third condition is somewhat more mysterious and, if comparing Law 2.9 to Law 2.8, does not seem to stem from the discrete evolution law. When condition (iii) is removed from Law 2.9, the evolution is under-constrained and may generate too many solutions as shown in Remark 4.18 below. It is shown in Remark 2.11 below to be a (formal) consequence of Law 2.8 in the case of MIL's.

Remark 2.10. According to condition (i), $\Gamma(0) \supset \Gamma_{0}$, thus, $E_{s}(\Gamma(0)) \geqslant E_{s}\left(\Gamma_{0}\right)$. But according to condition (ii), $E(\Gamma(0), 0)=E_{s}(\Gamma(0)) \leqslant E\left(\Gamma_{0}, 0\right)=E_{s}\left(\Gamma_{0}\right)$, thus, $E_{s}(\Gamma(0))=E_{s}\left(\Gamma_{0}\right)$, which implies, in view of Remark 2.1, that $\Gamma(0)=\Gamma_{0}$.

Remark 2.11. If $U(t)$ is a MIL, then,

$$
\Gamma^{\prime} \supset \Gamma \quad \text { and } \quad E\left(\Gamma^{\prime}, U(t)\right) \leqslant E(\Gamma, U(t)) \Rightarrow E\left(\Gamma^{\prime}, U(s)\right) \leqslant E(\Gamma, U(s)), \quad s \geqslant t .
$$

Indeed, according to Remark 2.7,

$$
\begin{aligned}
E(\Gamma, U(s))-E\left(\Gamma^{\prime}, U(s)\right) & =E(\Gamma, U(t))-E\left(\Gamma^{\prime}, U(t)\right) \\
+ & \left(s^{2}-t^{2}\right)\left(E_{d}\left(\Gamma, U_{0}\right)-E_{d}\left(\Gamma^{\prime}, U_{0}\right)\right) \geqslant E(\Gamma, U(t))-E\left(\Gamma^{\prime}, U(t)\right) .
\end{aligned}
$$

Then, if $\Gamma_{i}$ is a solution to the discrete evolution problem for $U_{i}$, a discretized MIL, (6) implies that $E\left(\Gamma_{i}, U(t)\right) \leqslant E\left(\Gamma_{i-1}, U(t)\right)$, for all $t \geqslant t_{i}$, from which it is immediately deduced that $E\left(\Gamma_{i}, U_{i}\right) \leqslant E\left(\Gamma_{j}, U_{i}\right), j \leqslant i$.

Condition (iii) in Law 2.9 is then formally obtained upon replacing $t_{i}$ by $t$ and $\Gamma_{k}$, $k \leqslant i$, by $\Gamma(s), s \leqslant t$. 


\section{A FEW ANALYTIC SOLUTIONS}

As a first test of the proposed formulation, two examples for which exact solutions can be computed are presented below: that of a three-dimensional (3-D) cylinder under uniaxial tension, and that of the tearing of a reinforcement in a cylindrical domain. The canonical one-dimensional (1-D) case is not discussed here; see Francfort and Marigo (to appear).

In both settings the loadings are assumed to be MIL's.

\subsection{Uniaxial tension of a 3-D cylinder}

Assume that $\Omega=S \times(0, L)$, with $S$ a smooth open connected domain of $\mathbb{R}^{2}$, that the material is isotropic and homogeneous with Young's modulus $E$, Poisson's ratio $v$, and fracture toughness $k(x)=\kappa$. Assume also that $\Gamma_{0}=\varnothing$, and that $u_{3}=0$ on $S \times\{0\}$ while $u_{3}=t$ on $S \times\{L\}$. In other words, the boundary conditions look, $a$ priori formally, like:

$$
\left\{\begin{array}{lll}
u_{3}=0 \quad \text { and } & \sigma_{13}=\sigma_{23}=0 & \text { on } S \times\{0\}, \\
u_{3}=t \quad \text { and } & \sigma_{13}=\sigma_{23}=0 & \text { on } S \times\{L\}, \\
\sigma \cdot n=0 & & \text { on } \partial S \times(0, L) .
\end{array}\right.
$$

Correspondingly, upon defining $\Omega_{d}=S \times(-\delta, L+\delta), \mathscr{C}(\Gamma, t)=\left\{v \in H^{1}\left(\Omega_{d} \backslash \Gamma ; \mathbb{R}^{3}\right)\right.$; $v_{3}=0$ in $S \times(-\delta, 0), v_{3}=t$ in $\left.S \times(L, L+\delta)\right\}$.

An easy computation would show that

$$
\begin{aligned}
& u(\varnothing, t)(x)=-\left(v t \frac{x_{1}}{L}+a_{1}\right) e_{1}-\left(v t \frac{x_{2}}{L}+a_{2}\right) e_{2}+t \frac{x_{3}}{L} e_{3}, \\
& \sigma(\varnothing, t)(x)=E \frac{t}{L} e_{3} \otimes e_{3},
\end{aligned}
$$

where $a_{1}$ and $a_{2}$ are two arbitrary constants that characterize the rigid transverse translations of the cylinder. The corresponding energies are

$$
E_{s}(\varnothing)=0, \quad E(\varnothing, t)=E_{d}(\varnothing, t)=\frac{1}{2} E \frac{t^{2}}{L}|S| .
$$

Let $\Gamma$ be an arbitrary crack and denote by $P(\Gamma)$ its projection onto $S ; P(\Gamma)$ is $\mathscr{H}^{2}$ measurable because it is compact; define $\Theta(\Gamma)=\mathscr{H}^{2}(P(\Gamma)) /|S|$. The energy of the body in an arbitrary cracked state is not explicitly computable but an estimate from below will prove sufficient for our purpose. This is the object of the following

Proposition 3.1. $E(\Gamma, t) \geqslant(1-\Theta(\Gamma)) E(\varnothing, t)+\Theta(\Gamma) \kappa|S|$.

Proof. Firstly,

$$
E_{s}(\Gamma, t) \geqslant \Theta(\Gamma) \kappa|S|,
$$

the inequality being strict unless $\mathscr{H}^{2}(P(\Gamma))=\mathscr{H}^{2}(\Gamma)$, that is unless $\Gamma$ is transverse. If $\Theta(\Gamma)=1$, (8) implies the proposition while, if not, i.e., if $P(\Gamma) \neq S$, 


$$
\begin{aligned}
E_{d}(\varnothing, t) & \geqslant E_{d}(\Gamma, t) \\
& =\inf _{v \in \mathscr{G}(\Gamma, t)} \frac{1}{2} \int_{\Omega_{\Gamma}} A \varepsilon(v) \cdot \varepsilon(v) \mathrm{d} x \\
& =\inf _{v \in \mathscr{G}(\Gamma, t)} \sup _{\tau \in\left[L^{2}\left(\Omega_{\Gamma}\right)\right]_{s}^{9}} \int_{\Omega_{\Gamma}}\left(\tau \cdot \varepsilon(v)-\frac{1}{2} A^{-1} \tau \cdot \tau\right) \mathrm{d} x \\
& \geqslant \sup _{\tau \in\left[L^{2}\left(\Omega_{\Gamma}\right)\right]_{s}^{9}} \inf _{v \in \mathscr{G}(\Gamma, t)} \int_{\Omega_{\Gamma}}\left(\tau \cdot \varepsilon(v)-\frac{1}{2} A^{-1} \tau \cdot \tau\right) \mathrm{d} x \\
& \geqslant \inf _{v \in \mathscr{C}(\Gamma, t)} \int_{\Omega_{\Gamma}}\left(\sigma(t) \cdot \varepsilon(v)-\frac{1}{2} A^{-1} \sigma(t) \cdot \sigma(t)\right) \mathrm{d} x,
\end{aligned}
$$

where

$$
\sigma(t)= \begin{cases}0, & \text { if } x \in P(\Gamma) \times(0, L), \\ \sigma(\varnothing, t), & \text { otherwise }\end{cases}
$$

is an admissible stress field. Now each element of $\mathscr{C}(\Gamma, t)$ has a third component with a well-defined trace on $S \backslash P(\Gamma) \times\{0$ (resp. $L)\}$, namely 0 (resp. $t$ ). Thus, since the only non-zero component of $\sigma(t)$ is its $3 \times 3$ component,

$$
\begin{aligned}
\int_{\Omega_{\Gamma}} \sigma(t) \cdot \varepsilon(v) \mathrm{d} x & =\int_{\Omega_{\Gamma}} \sigma_{33}(t) \cdot \frac{\partial v_{3}}{\partial x_{3}} \mathrm{~d} x \\
& =\int_{S \backslash \mathrm{P}(\Gamma) \times(0, \mathrm{~L})} \sigma_{33}(\varnothing, t) \cdot \frac{\partial u_{3}(\varnothing, t)}{\partial x_{3}} \mathrm{~d} x \\
& =\int_{S \mathrm{P}(\Gamma) \times(0, \mathrm{~L})} \sigma(\varnothing, t) \cdot \varepsilon(u(\varnothing, t)) \mathrm{d} x .
\end{aligned}
$$

Thus,

$$
\begin{aligned}
E_{d}(\Gamma, t) & \left.\geqslant \int_{S \backslash \mathrm{P}(\Gamma) \times(0, \mathrm{~L})} \sigma(\varnothing, t) \cdot \varepsilon(u(\varnothing, t))-1 / 2 A^{-1} \sigma(\varnothing, t) \cdot \sigma(\varnothing, t)\right) \mathrm{d} x \\
& =(1-\Theta(\Gamma)) E(\varnothing, t),
\end{aligned}
$$

which, together with (8), proves the proposition.

We are now in a position to prove the following

Proposition 3.2. The cylinder remains crack-free as long as the loading parameter $t$ remains strictly less than $t_{r}=[\sqrt{2(\kappa L / E)}]$. For $t>t_{r}$, a solution-crack consists in cutting the cylinder into two pieces along an arbitrary transverse section.

Furthermore this is the only type of solution in the class of cracks for which the infimum in (4) is attained.

Proof. According to (7), $E(\varnothing, t)<\kappa|S|$, as long as $t<t_{r}$, so that, by virtue of 
Proposition 3.1, $E(\Gamma, t)>E(\varnothing, t)$ for such times, except if $\Theta(\Gamma)=0$. But then, according to (9), (10), $E_{d}(\Gamma, t)=E_{d}(\varnothing, t)$, hence, if $\mathscr{H}^{2}(\Gamma) \neq 0, E(\Gamma, t)>E(\varnothing, t)$. Whenever $t>t_{r}, E(\varnothing, t)>\kappa|S|$, so that the same proposition implies that $E(\Gamma, t)>\kappa|S|$ except if $P(\Gamma)=S, \mathscr{H}^{2}(\Gamma)=|S|$, and $E_{d}(\Gamma, t)=0$. But then the displacement field that minimizes (4), when it exists, is necessarily a rigid body displacement $\left(\varepsilon(u)=0\right.$ on $\left.\Omega_{d} \backslash \Gamma\right)$ which also lies in $\mathscr{C}(\Gamma, t)$; this is impossible unless $\Gamma=\bar{S} \times\{z\}, z \in[0, L]$.

Remark 3.3. We do not know whether there might be solutions for $t>t_{r}$ which should be "spurious transverse cracks" without bulk energy such that $P(\Gamma)=S$ and $\mathscr{H}^{2}(\Gamma)=|S|$, but for which the infimum 0 in (4) is not achieved by any admissible displacement field.

\subsection{Tearing of a reinforcement}

A 3-D cylinder $\Omega=S \times(0, L)$ is investigated; its cross-section $S$ is an annulus of inner (resp. outer) radius $R_{i}$ (resp. $R_{0}$ ); it is made of a homogeneous, isotropic elastic material with Lamé coefficients $\lambda, \mu$ and toughness $\kappa$. A rigid, unbreakable reinforcement is bonded to the inner surface $\Sigma_{i}$ of the cylinder. Assume that the cylinder is initially crack-free $(\Gamma(t)=0, t<0)$, and that the following MIL is applied:

$$
u_{1}=u_{2}=\sigma_{33}=0 \quad \text { on } S \times\{0\}, S \times\{L\} ; \quad u=0 \quad \text { on } \Sigma_{0} ; u=t e_{3} \quad \text { on } \Sigma_{i},
$$

where $\Sigma_{0}$ denotes the outer surface of the cylinder. Once again these boundary conditions are formal and they have to be rewritten in terms of a set of kinematically admissible displacement fields

$$
\begin{gathered}
\mathscr{C}(\Gamma, t)=\left\{v \in H^{1}\left(\left(S \bigcup S_{0}(\delta) \bigcup S_{i}(\delta)\right) \times(-\delta, L+\delta) ; \mathbb{R}^{3}\right) ;\right. \\
v_{1}=v_{2}=0 \quad \text { on }\left(S \bigcup S_{0}(\delta) \bigcup S_{i}(\delta)\right) \times((-\delta, 0) \bigcup(L, L+\delta)), \\
\left.v=0 \quad \text { on } S_{0}(\delta) \times(0, L), \quad v=t e_{3} \quad \text { on } S_{i}(\delta) \times(0, L)\right\},
\end{gathered}
$$

where $S_{i}(\delta)$ is the annulus of inner radius $R_{i}-\delta$ and outer radius $R_{i}$ and $S_{0}(\delta)$ is the annulus with inner radius $R_{0}$ and outer radius $R_{0}+\delta$.

The result is the following

Proposition 3.4. The cylinder remains crack-free as long as

$$
t<t_{r} \equiv \sqrt{2 \frac{\kappa R_{i}}{\mu} \log \left(\frac{R_{0}}{R_{i}}\right)}
$$

while there is total debonding of the reinforcement from the inner surface of the cylinder as soon as $t>t_{r}$.

Proof. The proof is very similar to that of Proposition 3.2; it is sketched below.

The crack-free equilibrium fields are 


$$
u(\varnothing, t)=\frac{\log \left(r / R_{0}\right)}{\log \left(R_{i} / R_{0}\right)} t e_{3}, \quad \sigma(\varnothing, t)=\frac{t \mu}{2 r \log \left(R_{i} / R_{0}\right)}\left(e_{r} \otimes e_{3}+e_{3} \otimes e_{r}\right),
$$

with corresponding energies

$$
E(\varnothing, t)=E_{d}(\varnothing, t)=\pi \mu L \frac{t^{2}}{\log \left(R_{0} / R_{i}\right)} .
$$

A lower bound on the energy $E(\Gamma, t)$ is established by projecting $\Gamma$ onto the cylinder with radius $R_{i}$; if that projection is denoted by $P(\Gamma)$, we obtain $E(\Gamma, t) \geqslant(1-\Theta(\Gamma)) E(\varnothing, t)+\Theta(\Gamma) \kappa\left|\Sigma_{i}\right|$, where $\Theta(\Gamma)=\mathscr{H}^{2}(P(\Gamma)) /\left|\Sigma_{i}\right|$.

The result is then obtained through an argument identical to that developed in the proof of Proposition 3.2.

\section{GENERAL PROPERTIES OF THE PROPOSED MODEL}

This section investigates various characteristics of the crack evolution as predicted by the model and compares them with those foreseen by Griffith's theory of brittle fracture. Our analysis is, once again, restricted to MIL's, i.e., to boundary loadings of the form

$$
U(x, t)= \begin{cases}t U_{0}(x), & t \geqslant 0 \\ 0, & t<0\end{cases}
$$

\subsection{Crack initiation and failure of the sample}

We propose to demonstrate in this subsection that the model predicts crack initiation in a crack-free environment, in striking contrast with Griffith's theory; we also evidence eventual mechanical failure under ever-increasing loadings. Although our main setting (the only one we discussed as of yet) is the no-contact case, we will, in this subsection, express our results independently of the no-contact hypothesis, so as to demonstrate their applicability to the more general settings alluded to in Subsection 5.1.

To this effect we introduce the set of bulk-free states for a given loading $U$, namely

$$
\mathscr{F}(U)=\left\{\Gamma ; E_{d}\left(\Gamma, U_{0}\right)=0\right\} .
$$

Note that, if $\Gamma$ is an element of $\mathscr{F}(U)$, then any $\Gamma^{\prime} \supset \Gamma$ is also an element of $\mathscr{F}(U)$.

Remark 4.1. There are many examples of sets in $\mathscr{F}(U)$. For example,

(i) $\partial_{d} \Omega$ belongs to $\mathscr{F}(U)$, since, in such a case, $u=0$ is a kinematically admissible field,

(ii) Any $\Gamma \supset \partial_{d} \Omega$ belongs to $\mathscr{F}(U)$,

(iii) In the 3-D traction problem investigated in Subsection 3.1, any crack that splits the cylinder into connected components such that $S \times\{0\}$ and $S \times\{L\}$ do not live in the closure of the same component belongs to $\mathscr{F}(U)$.

Note however that, if unilateral conditions are to replace the no-contact condition 
adopted in most of this study (cf Remark 2.4 and Subsection 5.1 below), then $\mathscr{F}(U)$ might be empty.

Let us denote by $t_{i}$ the initiation time, i.e., the time when the crack first expands from its original site,

$$
t_{i}=\sup \left\{t ; \Gamma(t)=\Gamma_{0}\right\} .
$$

Recall that $\Gamma_{0}$ may be empty. If $t_{i}=0$, the sample experiences crack expansion as soon as it is loaded, while, if $t_{i}=\infty$, no further cracking will appear throughout the loading history. The following simple remark holds true:

Proposition 4.2. Assume that $\mathscr{F}(U) \neq \varnothing$ (which is true in the no-contact case). Then,

$$
t_{i}=\infty \text { iff } \Gamma_{0} \in \mathscr{F}(U) .
$$

Proof. If $\Gamma_{0} \in \mathscr{F}(U)$, then $\Gamma(t)\left(\supset \Gamma_{0}\right) \in \mathscr{F}(U)$, thus, $E_{d}\left(\Gamma(t), U_{0}\right)=0$. According to (iii) in Law 2.9, $E_{s}(\Gamma(t)) \leqslant E_{s}\left(\Gamma_{0}\right)$. Since however $\Gamma(t) \supset \Gamma_{0}$, the surface energies are equal and, recalling Remark 2.1, $\Gamma(t)=\Gamma_{0}$.

Conversely, if $t_{i}=\infty$, then $\Gamma(t)=\Gamma_{0}, t>0$, thus, by virtue of (ii) in Law 2.9,

$$
t^{2} E_{d}\left(\Gamma_{0}, U_{0}\right)+E_{s}\left(\Gamma_{0}\right) \leqslant t^{2} E_{d}\left(\Gamma, U_{0}\right)+E_{s}(\Gamma), \quad \text { for all } \Gamma \supset \Gamma_{0} .
$$

Take $\Gamma=\Gamma_{0} \bigcup \Gamma^{\prime}$ with $\Gamma^{\prime} \in \mathscr{F}(U)$, so that $\Gamma \in \mathscr{F}(U)$. Then

$$
t^{2} E_{d}\left(\Gamma_{0}, U_{0}\right)+E_{s}\left(\Gamma_{0}\right) \leqslant E_{s}(\Gamma), t \geqslant 0,
$$

which is only possible if $E_{d}\left(\Gamma_{0}, U_{0}\right)=0$.

Remark 4.3. An immediate consequence of the above proposition is that, if, in the no-contact case, $\Gamma_{0}=\varnothing$ (no preexisting crack) and $U_{0}$ is not a rigid displacement, a crack will always appear in finite time. The reader is, once again, reminded that crack initiation is a sore point in the classical theories of brittle fracture.

We now investigate the asymptotics of fracture. Define the state of least bulk energy,

$$
E_{d}^{\min }=\inf \left\{E_{d}\left(\Gamma, U_{0}\right) ; \Gamma \supset \Gamma_{0}\right\},
$$

and recall that, if $\mathscr{F}(U)=\varnothing, E_{d}^{\min }=0$ (the no-contact case). Then,

\section{Proposition 4.4.}

(i) $\lim _{t \rightarrow \infty} E_{d}\left(\Gamma(t), U_{0}\right)=E_{d}^{\mathrm{min}}$,

(ii) If there exists a finite first time $t_{f}$ such that $E_{d}\left(\Gamma\left(t_{f}\right), U_{0}\right)=E_{d}^{\min }$, then $\Gamma(t)=\Gamma\left(t_{f}\right)$, $t \geqslant t_{f}$.

The time $t_{f}$ is referred to as the failure time for the sample.

Proof. If $\left\{\Gamma_{n}\right\}_{n}$ is a minimizing sequence for (12), $\left\{\Gamma_{n} \cup \Gamma(t)\right\}_{n}, t \geqslant 0$ is one as well in view of (ii) in Remark 2.7. Therefore, (ii) in Law 2.9 implies that

$$
\begin{aligned}
t^{2} E_{d}\left(\Gamma(t), U_{0}\right)+E_{s}(\Gamma(t)) & \leqslant t^{2} E_{d}\left(\Gamma_{n} \bigcup \Gamma(t), U_{0}\right)+E_{s}\left(\Gamma_{n} \bigcup \Gamma(t)\right) \\
& \leqslant t^{2} E_{d}\left(\Gamma_{n}, U_{0}\right)+E_{s}\left(\Gamma_{n}\right)+E_{s}(\Gamma(t)) .
\end{aligned}
$$

Thus, dividing by $t^{2}$ in the above inequality, then letting $t$ and $n$ tend to $\infty$ yields (i). 
If $E_{d}\left(\Gamma\left(t_{f}\right), U_{0}\right)=E_{d}^{\min }$ for some $t_{f}$, then (iii) in Law 2.9 implies that $E_{s}(\Gamma(t))$ $\leqslant E_{s}\left(\Gamma\left(t_{f}\right)\right), t \geqslant t_{f}$, and, since $\Gamma(t) \supset \Gamma\left(t_{f}\right), t \geqslant t_{f}$, (ii) is established.

Remark 4.5. In the no-contact case, the previous result demonstrates that, under everincreasing loadings, the crack will expand until a bulk-free state is reached (a state without elastic energy), which we will view as synonymous to a state of mechanical failure. This, together with Remark 4.3, provides a rather complete picture of crack evolution in our main setting, namely that of no-contact : a crack will appear in finite time and will expand until mechanical failure in finite or infinite time.

Actually, an estimate of times $t_{i}$ and $t_{f}$ can be derived.

Proposition 4.6. If $\mathscr{F}(U) \neq \varnothing$ and if $\Gamma_{0} \notin \mathscr{F}(U)$, then

$$
0 \leqslant t_{i} \leqslant \sqrt{\frac{\inf \left\{E_{s}\left(\Gamma \backslash \Gamma_{0}\right) ; \Gamma \supset \Gamma_{0}, \Gamma \in \mathscr{F}(U)\right\}}{E_{d}\left(\Gamma_{0}, U_{0}\right)}} \leqslant t_{f} \leqslant \infty .
$$

Proof. Take $\Gamma \supset \Gamma_{0}, \Gamma \in \mathscr{F}(U)$. Then, for every $t<t_{i}$, (ii) of Law 2.9 implies that

$$
t^{2} E_{d}\left(\Gamma_{0}, U_{0}\right) \leqslant E_{s}(\Gamma)-E_{s}\left(\Gamma_{0}\right)=E_{s}\left(\Gamma \backslash \Gamma_{0}\right),
$$

hence the first inequality upon letting $t$ tend to $t_{i}$ and infimizing in $\Gamma$.

If $t_{f}=\infty$ the second inequality is trivial, while otherwise, for $t>t_{f}$, (iii) of Law 2.9 yields

$$
E_{s}(\Gamma(t)) \leqslant t^{2} E_{d}\left(\Gamma_{0}, U_{0}\right)+E_{s}\left(\Gamma_{0}\right) .
$$

But then $\Gamma(t) \in \mathscr{F}(U)$ and $\Gamma(t) \supset \Gamma_{0}$, so

$$
\inf \left\{E_{s}\left(\Gamma \backslash \Gamma_{0} ; \Gamma \supset \Gamma_{0}, \Gamma \in \mathscr{F}(U)\right\} \leqslant E_{s}(\Gamma(t))-E_{s}\left(\Gamma_{0}\right) \leqslant t^{2} E_{d}\left(\Gamma_{0}, U_{0}\right),\right.
$$

and the result is obtained upon letting $t$ tend to $t_{f}$.

Remark 4.7.

(i) Although $\Gamma(0)=\Gamma_{0}$, it is possible for $t_{i}$ to be 0 (see Section 4.4 below),

(ii) Whenever $0<t_{i}=t_{f}<\infty$ the crack evolution is as follows:

$$
\Gamma(t)= \begin{cases}\Gamma_{0} & \text { if } t<t_{i}=t_{f}, \\ \Gamma_{f} & \text { if } t>t_{i}=t_{f},\end{cases}
$$

where $\Gamma_{f}$ is an element such that $E_{d}^{\min }=E_{d}\left(\Gamma_{f}, U_{0}\right)$. Such is the case in the two examples developed in Section 3,

(iii) We do not know of any case where $t_{i}<t_{f}=\infty$.

\section{2. $\quad$ Griffith vs energy minimization}

This subsection is devoted to a comparison between Griffith's theory and our model. The framework is that of no-contact 2-D elasticity. The following is assumed throughout this subsection as well as the next one:

Predefined crack path: During a MIL, the crack path is a rectifiable curve $\Gamma$ of the 
finite length $L$ with origin $x_{0}$ and end point $x_{L}$. It is parameterized by its arc-length $x(s)$ with $x(0)=x_{0}$. Define

$$
\Gamma(l) \equiv \Gamma_{0} \bigcup\{x(s) ; 0 \leqslant s \leqslant l\} .
$$

Then the bulk and surface energies become functions of $l$, namely,

$$
\begin{aligned}
F(l) & \equiv E_{d}\left(\Gamma(l), U_{0}\right), \\
H(l) & \equiv E_{s}(\Gamma(l))-E_{s}\left(\Gamma_{0}\right) .
\end{aligned}
$$

These functions are, respectively, monotonically decreasing and increasing with respect to $l$; we also assume that they are as smooth as needed. Finally we denote by $L_{0}$ the length of $\Gamma_{0}$.

It now remains to investigate the trajectory of the crack along its path, i.e., the function $t \rightarrow l(t)$. The first result concerns progressive crack evolution.

Proposition 4.8. If $l(t)$ is an absolutely continuous function of $t$, then Griffith's law of crack evolution is satisfied. In other words,

(i) $l(0)=0$,

(ii) $\dot{l}(t) \geqslant 0$,

(iii) $-t^{2}(\mathrm{~d} F / \mathrm{d} l)(l(t)) \leqslant k(x(l(t)))$,

(iv) $\dot{l}(t)=0$ if the inequality is strict in (iii).

Proof. Items (i) and (ii) are obvious. Item (iii) is obtained by applying (ii) of Law 2.9 at time $t$, with $\Gamma(l(t)+h), h \geqslant 0$ as test crack, while item (iv) is obtained by applying (iii) of Law 2.9 at time $t$ with $s=t-h$.

Remark 4.9. Item (iii) in the above proposition is precisely Griffith's criterion, since $-t^{2}(\mathrm{~d} F / \mathrm{d} l)(l(t))$ represents the energy release rate. Item (iv) is the classical assumption that propagation will not take place unless the energy release rate becomes critical.

If now $l(t)$ experiences a jump at time $t_{0}$, the following proposition holds:

Proposition 4.10. If at time $t_{0}, l^{-}\left(t_{0}\right) \neq l^{+}\left(t_{0}\right)$, then,

$$
-t_{0}^{2}\left(F\left(l^{+}\left(t_{0}\right)\right)-F\left(l^{-}\left(t_{0}\right)\right)\right)=H\left(l^{+}\left(t_{0}\right)\right)-H\left(l^{-}\left(t_{0}\right)\right) .
$$

Proof. Apply (ii) of Law 2.9 to time $t_{0}-h$ with $\Gamma\left(l\left(t_{0}+h\right)\right)$ as test crack; then,

$$
\left(t_{0}-h\right)^{2} F\left(l\left(t_{0}-h\right)\right)+H\left(l\left(t_{0}-h\right)\right) \leqslant\left(t_{0}-h\right)^{2} F\left(l\left(t_{0}+h\right)\right)+H\left(l\left(t_{0}+h\right)\right),
$$

and passing to limit in $h$ yields one inequality. The other inequality is obtained by applying (iii) of Law 2.9 to $t=t_{0}+h$, taking as previous instant $t=t_{0}-h$, so that

$$
\left(t_{0}+h\right)^{2} F\left(l\left(t_{0}+h\right)\right)+H\left(l\left(t_{0}+h\right)\right) \leqslant\left(t_{0}+h\right)^{2} F\left(l\left(t_{0}-h\right)\right)+H\left(l\left(t_{0}-h\right)\right),
$$

which yields the result upon passing to the limit in $h$.

Remark 4.11. The above condition, reminiscent of Rankine-Hugoniot conditions across a shock in conservation laws, or rather of a kind of Maxwell condition in the theory of phase transition, merely expresses global energy conservation during a brutal crack jump. It can be reinterpreted, in a homogeneous medium, as a propagation criterion for the mean energy release rate, i.e., 


$$
\frac{-t_{0}^{2}}{l^{+}\left(t_{0}\right)-l^{-}\left(t_{0}\right)} \int_{l^{-}\left(t_{0}\right)}^{l^{+}\left(t_{0}\right)} \frac{\mathrm{d} F}{\mathrm{~d} l}(l) \mathrm{d} l=k .
$$

Remark 4.12. In Hashin (1996), the jump condition is a priori postulated as a generalized Griffith's law. It cannot however be sufficient as such to predict when and how such a jump will occur, which, by contrast, is within reach of our formulation.

\subsection{Progressive evolution or brutal evolution}

In this subsection, we investigate the circumstances that preside over the brutal vs progressive character of crack growth. We remind the reader of our assumption of "predefined crack path", as detailed in the previous subsection. To this end, we reparameterize the crack path in terms of its surface energy by setting

$$
\lambda=H(l), \quad \hat{F}(\lambda)=F\left(H^{-1}(\lambda)\right) ;
$$

such a change is licit since, in view of Remark $2.1, H$ is a continuous strictly increasing function of $\lambda$. Note that $\hat{F}$ is a monotonically decreasing function of $\lambda$ in view of (13).

In the present context the evolution law described in Law 2.9 (partially) translates into

Law 4.13. The crack trajectory $t \rightarrow \lambda(t)$ must be such that

(i) $\lambda(t)$ is a monotonically increasing function of $t$,

(ii) $t^{2} \hat{F}(\lambda(t))+\lambda(t) \leqslant t^{2} \hat{F}(\lambda)+\lambda, \lambda \geqslant \lambda^{-}(t)$,

(iii) $t^{2} \hat{F}(\lambda(t))+\lambda(t) \leqslant t^{2} \hat{F}(\lambda(s))+\lambda(s), s \leqslant t$.

Note that item (ii) in the above law is a rather degenerate offspring of its parent in Law 2.9 because it only envisions crack evolutions along the predefined crack path.

The brutal vs progressive character of crack growth is then intimately linked to the convexity properties of $\hat{F}$. Specifically, we obtain the following

Proposition 4.14. If $\hat{F}$ is continuous, then $\lambda(t)$ lives, for each $t$, among the minimizers of $t^{2} \hat{F}(\lambda)+\lambda$ on $[0, H(L)]$.

Proof. We define $\lambda(t)$ as (one of) the minimizer(s) of $t^{2} \hat{F}(\lambda)+\lambda$ on $[0, H(L)]$. Note that the minimizers increase with $t$ so that $\lambda(t)$ increases with $t$. At time $t=0, \lambda(0)=0$. Conditions (ii) and (iii) in Law 4.13 are trivially met. Thus, such a $\lambda(t)$ is always a solution.

Conversely, if $\lambda(t)$ is a solution, then

$$
t^{2} \hat{F}(\lambda(t))+\lambda(t) \leqslant t^{2} \hat{F}(\lambda)+\lambda, \quad \lambda \geqslant \lambda^{-}(t),
$$

according to (ii) in Law 4.13. Thus, it suffices to extend this inequality to the interval $\left[0, \lambda^{-}(t)\right]$. Now $\lambda(t)$ has, at most, a countable number of discontinuities on $\left[0, \lambda^{-}(t)\right]$; label them $\left\{t_{p}\right\}$ and the corresponding jumps $\left[\lambda_{p}^{-}, \lambda_{p}^{+}\right]$(so that $\lambda\left(t_{p}\right)$ lives somewhere within $\left.\left[\lambda_{p}^{-}, \lambda_{p}^{+}\right]\right)$. For $\lambda \notin \bigcup_{p}\left[\lambda_{p}^{-}, \lambda_{p}^{+}\right]$, the inequality is satisfied by virtue of condition (iii) in Law 4.13. Take $t=t_{p}+h, h>0$. Then, passing to the limit as $h$ tends to 0 in (iii) of Law 4.13, we obtain the inequality for $\lambda=\lambda_{p}^{+}$. Recalling Proposition 4.10 which remains valid at any jump of $\lambda$, as well as (ii) of Law 4.13, we obtain 


$$
t_{p}^{2} \hat{F}\left(\lambda_{p}^{-}\right)+\lambda_{p}^{-}=t_{p}^{2} \hat{F}\left(\lambda_{p}^{+}\right)+\lambda_{p}^{+} \leqslant t_{p}^{2} \hat{F}(\lambda)+\lambda, \quad \lambda \geqslant \lambda_{p}^{-} .
$$

Then, by virtue of the decreasing character of $\hat{F}$, we obtain, for any $\lambda \in\left[\lambda_{p}^{-}, \lambda_{p}^{+}\right]$,

$$
\begin{aligned}
t^{2} \hat{F}\left(\lambda_{p}^{+}\right)+\lambda_{p}^{+} & =t_{p}^{2} \hat{F}\left(\lambda_{p}^{+}\right)+\lambda_{p}^{+}+\left(t^{2}-t_{p}^{2}\right) \hat{F}\left(\lambda_{p}^{+}\right) \\
& \leqslant t_{p}^{2} \hat{F}\left(\lambda_{p}^{+}\right)+\lambda_{p}^{+}+\left(t^{2}-t_{p}^{2}\right) \hat{F}(\lambda) \\
& \leqslant t_{p}^{2} \hat{F}(\lambda)+\lambda+\left(t^{2}-t_{p}^{2}\right) \hat{F}(\lambda) \\
& =t^{2} \hat{F}(\lambda)+\lambda
\end{aligned}
$$

which proves the inequality for $\lambda \in\left[\lambda_{p}^{-}, \lambda_{p}^{+}\right]$.

Whenever $\hat{F}$ exhibits decent convexity properties, the previous proposition permits to precisely determine the evolution of the crack, i.e., $\lambda(t)$. We obtain the following Corollary 4.15.

(i) If $\hat{F}$ is $\mathscr{C}^{1}$ and strictly convex, then $\lambda(t)$ is given by

$$
\lambda(t)= \begin{cases}0 & \text { if } 0 \leqslant t \leqslant t_{i}, \\ \hat{F}^{\prime-1}\left(-\frac{1}{t^{2}}\right) & \text { if } t_{i} \leqslant t \leqslant t_{L}, \\ H(L) & \text { if } t_{L} \leqslant t,\end{cases}
$$

with

$$
\begin{aligned}
t_{i} & =\sqrt{1 /\left(-\hat{F}^{\prime}(0)\right)}, \\
t_{L} & =\sqrt{1 /\left(-\hat{F}^{\prime}(H(L))\right)} ;
\end{aligned}
$$

in other words, the crack growth is progressive,

(ii) If $\hat{F}$ is concave, then $\lambda(t)$ is given by

$$
\lambda(t)= \begin{cases}0 & \text { if } 0 \leqslant t<t_{i}=t_{L} \\ H(L) & \text { if } t_{i}=t_{L}<t\end{cases}
$$

with

$$
t_{L} \equiv \sqrt{H(L) /(\hat{F}(0)-\hat{F}(H(L)))} ;
$$

in other words, the crack growth is brutal.

Proof. If $\hat{F}$ is convex and $\mathscr{C}^{1}, t^{2} \hat{F}(\lambda)+\lambda$ attains its minimum at $\lambda=0$ if $t^{2} \hat{F}^{\prime}(0)+1 \geqslant 0$, at $H(L)$ if $t^{2} \hat{F}^{\prime}(H(L))+1 \leqslant 0$, and otherwise at $\lambda$ such that $t^{2} \hat{F}^{\prime}(\lambda)+1=0$; application of Proposition 4.14 then yields (i). If however $\hat{F}$ is concave, $t^{2} \hat{F}(\lambda)+\lambda$ can only attain its minimum at $\lambda=0$ if $t<t_{L}$, and at $\lambda=H(L)$ if $t>t_{L}$; application of Proposition 4.14 then yields (ii).

Remark 4.16. Whenever $\hat{F}$ is convex but grows linearyly on, say $\left[\lambda_{1}, \lambda_{2}\right]$, the crack will experience a jump at time $t=\sqrt{1 / \hat{F}^{\prime}}\left(\lambda_{1}\right)=\sqrt{1 / \hat{F}\left(\lambda_{2}\right)}$. 
Remark 4.17. Whenever $\hat{F}$ is not convex, the minima of $t^{2} \hat{F}(\lambda)+\lambda$ will be those of $t^{2} \hat{F}^{* *}(\lambda)+\lambda$, where $\hat{F}^{* *}$ denotes the convexification of $\hat{F}$. Thus, the crack evolution will be progressive on the strictly convex parts of the graph of $\hat{F}^{* *}$ and brutal on the linear parts of that graph.

Remark 4.18. Assume that (iii) of Law 4.13 [or a fortiori (iii) of Law 2.9] is abandoned. Assume that $\hat{F}$ is strictly convex and consider a time $t \in\left(t_{i}, t_{L}\right)$ (cf corollary 4.15). Then, if $\lambda \in[0, \lambda(t)], \lambda$ satisfies (ii) of Law 4.13 , and we can choose many monotonically increasing trajectories $\lambda(t)$ that satisfy (ii) besides $\lambda(t)$. Thus (iii) can be viewed as a selection criterion among all those trajectories that will force the minimal trajectory $\lambda(t)$.

If comparing the above results to those yielded by Griffith's theory, it is easily seen that, in the strictly convex case, the results are identical, but that, in the concave case, Griffith's criterion will actually predict initiation at time $t_{G}=\sqrt{1 /\left(-\hat{F}^{\prime}(0)\right)}$ (cf (iv) of Proposition 4.8), but then, the criterion will not be met for $t>t_{G}$ by any $\lambda$. The crack is then baptized "unstable" and henceforth untractable. Note also that the predicted initiation time $t_{G}$ is longer than that, namely $t_{L}$, obtained through our formulation. If, in general, $\hat{F}^{\prime}(0)=0$, Griffith's theory will not permit crack initiation (cf (iv) of Proposition 4.8), whereas ours will generate brutal growth, as is immediately deduced from the preceding results.

\subsection{The role played by singularities; the 2-D case}

In Griffith's theory, displacement and stress singularities play an essential role; in a 2-D setting, the $\sqrt{r}$-singularity of the displacement field ensures the finiteness of the energy release rate $G$, while Irwin's formula permits the computation of $G$ in terms of the stress intensity factors. As will be seen below, singularities do play an important role in our formulation as well.

We assume throughout this subsection that, if $\Gamma_{0}$ is the initial crack (maybe empty), then the displacement field $u_{0} \equiv u\left(\Gamma_{0}, U_{0}\right)$, corresponding to elastic equilibrium on $\Omega \backslash \Gamma_{0}$ for the loading $U_{0}$, is of the form

$$
u_{0}(x)=\sum_{p=1}^{n} r_{p}^{\alpha} v_{p}\left(\theta_{p}\right)+\hat{u}(x)
$$

where the $\left(r_{p}, \theta_{p}\right)$ are polar coordinates with poles $\left\{x_{p}\right\}_{p=1, n}$, the singular points of $u_{0}$, and $0<\alpha_{p}<1$. We also assume that the toughness is constant, equal to $\kappa_{p}$ in a neighborhood of $x_{p}$. Note that the restriction on $\alpha_{p}$ ensures the finiteness of the bulk energy and the singular character of $u_{0}$, that is $u_{0} \notin H^{2}$, or, in other words, its second order derivatives are not square integrable. The points $x_{p}$ could be crack tips, points on the boundary that experience a change in boundary condition type, or non-smooth points of the boundary.

We finally assume, when needed, the following restriction on crack paths:

Predefined incremental crack path $(P I C P)$ : Crack growth for small times only takes place along $n+1$ rectifiable curves $\left\{\Gamma_{l_{p}}\right\}_{p=1, n+1}$ of finite length $l_{p}$, where $\Gamma_{l_{p}}, p \leqslant n$ is 
located within a small neighborhood of the corresponding singular point $x_{p}$, and $\Gamma_{l_{n+1}}$ is located within a small neighborhood of a non-singular point.

We propose to investigate the brutal/progressive character of the crack growth at initiation time $t_{i}$. To this effect, it is imperative to know an expansion of the bulk energy in terms of the length of a small add-defect. In Leguillon (1990a), an asymptotic expansion of that energy in terms of the added length is proposed, provided that $E_{d}\left(\Gamma_{0}, U_{0}\right)>0$, namely,

$$
E_{d}\left(\Gamma_{0} \cup \Gamma_{l}, U_{0}\right)=E_{d}\left(\Gamma_{0}, U_{0}\right)-\sum_{p=1}^{n+1}\left\{K_{p} l_{p}^{2 \alpha_{p}}+o\left(l_{p}^{2 \alpha_{p}}\right)\right\},
$$

where $K_{p}$ is a positive (except maybe $K_{n+1}$ which may take the value 0 ) factor which depends on the shape of the defect, on the strength of the singularity at $x_{p}$, but not on the length $l_{p}$ of the defect $\Gamma_{l_{p}}\left(\Gamma_{l}=\bigcup_{p=1}^{n+1} \Gamma_{l_{p}}\right)$. In (14) the add-crack is assumed to be living in a neighborhood of the singular point $x_{p}$; finally this expansion remains valid for the add-crack $\Gamma_{l_{n+1}}$ with $\alpha_{n+1}=1$.

We are now in a position to establish the following

Proposition 4.19. In the above described context,

(i) If at least one $x_{p}$ exhibits a strong singularity $\left(\alpha_{p}<1 / 2\right)$, then the crack growth will be progressive, with zero initiation time, and, under the PICP hypothesis, the length of the add-crack corresponding to $x_{p}$ grows like $t^{2 /\left(1-2 \alpha_{p}\right)}$,

(ii) If, under the PICP hypothesis, all points $x_{p}$ exhibit a weak singularity $\left(\alpha_{p}>1 / 2\right)$, then the crack growth will be brutal with a non-zero, finite initiation time,

(iii) If, under the PICP hypothesis, the most singular points exhibit a $\sqrt{r}$-singularity, then the crack growth will have a non-zero, finite initiation time,

(iv) If, under the PICP hypothesis, there are no singular points, then, either there is no crack growth, or the crack growth will be brutal with a non-zero, finite initiation time.

Proof. To prove (i), assume that $t_{i}>0$. Take $0<t<t_{i}$, and consider, near $x_{p}$, an addcrack of length $\bar{l}_{p}(t)$, with

$$
\bar{l}_{p}(t)=\left(2 \alpha_{p} K_{p} t^{2} / \kappa_{p}\right) \frac{1}{1-2 \alpha_{p}} .
$$

Then, by virtue of (14),

$$
E\left(\Gamma_{0} \bigcup \Gamma_{\bar{l}_{p}(t)}, t U_{0}\right)=E\left(\Gamma_{0}, t U_{0}\right)-\left(\frac{1}{2 \alpha_{p}}-1\right) \kappa_{p} \bar{l}_{p}(t)+o\left(\bar{l}_{p}(t)\right)<E\left(\Gamma_{0}, t U_{0}\right),
$$

if $t$ is small enough, which contradicts (ii) of Law 2.9. Thus, $t_{i}=0$. Now, from (iii) of that law, $E_{d}\left(\Gamma(t), t U_{0}\right)+E_{s}(\Gamma(t)) \leqslant E_{d}\left(\Gamma_{0}, t U_{0}\right)+E_{s}\left(\Gamma_{0}\right)$, and, upon letting $t$ tend to 0 , this inequality yields $E_{s}\left(\bigcap_{t>0} \Gamma(t)\right) \leqslant E_{s}\left(\Gamma_{0}\right)$, which, since $\bigcap_{t>0} \Gamma(t) \supset \Gamma_{0}$ implies that $\Gamma(t) \searrow \Gamma_{0}$ as $t$ tends to 0 . Now, under the PICP hypothesis, the crack for small $t$ is of the form $\Gamma(t) \backslash \Gamma_{0}=\bigcup_{p=1}^{n+1} \Gamma_{l_{p}(t)}$, where $\Gamma_{l_{p}(t)} \subset \Gamma_{l_{p}}$. Setting $\alpha_{n+1}=1$, we get, in view of (14), 


$$
E\left(\Gamma(t), t U_{0}\right)=E\left(\Gamma_{0}, t U_{0}\right)+\sum_{p=1}^{n+1}\left[-t^{2} K_{p} l_{p}^{2 \alpha_{p}}(t)+\kappa_{p} l_{p}(t)+o\left(l_{p}(t)\right)\right]
$$

On the one hand, since $\Gamma(t) \searrow \Gamma_{0}$ as $t$ tends to $0, l_{p}(t) \searrow 0$ as well. On the other hand, Proposition 4.14 forces $l_{p}(t)$ to minimize

$$
-t^{2} K_{p} l^{2 \alpha_{p}}+\kappa_{p} l+o(l) .
$$

Whenever $\alpha_{p}<1 / 2$, the minimum is attained for $l_{p}(t)=\bar{l}_{p}(t)$ given in (15), whereas, if $\alpha_{p}>1 / 2$, the minimum is 0 . This proves (i).

In the setting of (ii), $t_{i}$ cannot be 0 , otherwise the argument developed in the proof of (i) would lead to $l_{p}(t)=0$, for $t$ close to 0 , i.e., to $\Gamma(t)=\Gamma_{0}$ for such $t$ 's, which contradicts $t_{i}=0$. Further $t_{i}<\infty$ according to Proposition 4.2. The argument developed in the proof of (i) also applies near $t_{i}$. Thus, if the crack evolution were progressive (i.e., if $\Gamma(t) \backslash \Gamma_{0}$ as $t$ tends to $t_{i}$ ), then necessarily $l_{p}(t) \searrow 0$ as $t$ tends to $t_{i}$, but, since all $\alpha_{p}>1 / 2$, the minimum in (16) is 0 , i.e., all $l_{p}(t)$ 's would stay 0 in a neighborhood of $t_{i}$, which is impossible. This proves (ii).

Items (iii) and (iv) are proved using identical arguments. Note that when $\alpha_{p}=1 / 2$ we cannot conclude on the value of the minimum in (16), hence on the brutal vs progressive character of the crack growth.

Remark 4.20. In the presence of a weak singularity $(\alpha>1 / 2)$ Griffith's theory will predict that no further cracking will occur, because the energy release rate is 0 , so that growth is not possible. But, in our formulation, this does not occur because crack growth will be brutal in such a case.

Remark 4.21. A similar analysis could be performed in the case where only a "tiny" connecting arc remains between two connected components of the domain. A different expansion derived by Leguillon (1990b) would be used and the conclusion would be that that arc would break brutally (i.e., in our language, that $\bigcup_{t<t_{f}} \Gamma(t) \neq \Gamma\left(t_{f}\right)$.

Remark 4.22. In view of the results of Proposition 4.19 and of the previous remark, it is seen that brutal growth is indeed a likely event, as demonstrated in the explicit computations of Section 3.

\section{EXTENSIONS AND OBSTACLES}

In a first subsection, two extensions of the model are briefly described; one permits to reconcile Griffith's approach to Barenblatt's with minor changes to our formulation; the other one pertains to lip contact along a crack and proposes a somewhat more realistic model. In a second subsection, various issues are raised about the model, and most notably its current inadequacy at confronting force loads.

\subsection{More realistic models}

As alluded to in Remark 2.2, the surface energy could be modified, so as to incorporate the effect of toughness anisotropy. Specifically, (1) can be replaced by 


$$
E_{s}(\Gamma)=\int_{\Gamma} k(x, v(x)) \mathrm{d} \mathscr{H}^{N-1}(x),
$$

provided that $\Gamma$ is an $N-1$-rectifiable set, so that its normal is well defined. As mentioned before, we cannot anymore allow for arbitrary closed sets of finite $\mathscr{H}^{N-1}$ measure. This restriction, which may appear as unwelcome, is actually no restriction at all, provided that the global minimization problem described in Section 2 be reformulated as a one-field minimization problem over kinematically admissible displacement fields that live in a bounded variation type space $(S B V$ or $S B D)$, because the jump set of such functions (which then replaces the crack) is then $N-1$-rectifiable (see Section 6). As stated in the introduction, we do not want to dwell in this study upon the many and complex mathematical questions that underlie the proposed model and refer the interested reader to the bibliography suggested in Section 6.

A further extension of (1) is

$$
E_{s}(\Gamma, u)=\int_{\Gamma} k(x, v(x),[u(x)] \cdot v(x)) \mathrm{d} \mathscr{H}^{N-1}(x),
$$

where $[u(x)]$ denotes the jump in the displacement field across a point $x$ of $\Gamma$. The surface energy is now a function of the displacement field $u$ and the minimization over kinematically admissible displacement fields has to be performed for the whole energy, rather than solely for $E_{d}$. Once again, such a model requires a well defined normal vector, as well as well-defined traces of the displacement field $u$ across the lips of the crack, and, once again, bounded variation type spaces come in handy and provide a meaningful interpretation of (18); cf Section 6. Note that the dependence of $k(x, p, s)$ upon $s$ has to exhibit concavity and monotonicity properties for the mathematical formulation in a bounded variation type space to make sense (see Ambrosio, 1989). The purpose of such an energy is twofold. On the one hand, it incorporates within our formulation fracture energies of Barenblatt's type, see Barenblatt (1962), which, some will argue, is a more realistic model for the surface energy associated to a crack. On the other hand, upon choosing

$$
k(x, p, s)=\infty, \quad \text { if } s<0,
$$

it allows (at least formally) for the consideration of lip interpenetration. Specifically, such an energy bans, as energetically unfavorable, those configurations where matter would interpenetrate at the crack site. Such a ban can also be imposed as a restriction on the set of kinematically admissible displacements $\mathscr{C}(\Gamma, U)$, which then becomes in lieu of (2),

$$
\mathscr{C}(\Gamma, U)=\left\{v \in H^{1}\left(\Omega_{d} \backslash \Gamma ; \mathbb{R}^{N}\right) ; v=U_{d} \text { in } \Omega_{d} \backslash \bar{\Omega},[v] \cdot v \geqslant 0 \text { on } \Gamma\right\} .
$$

Of course, such a definition is meaningless unless the trace of $v$ is adequately defined on $\Gamma$. We refer the concerned reader to the referenced literature on $B V(B D)$-spaces, for instance Evans and Gariepy (1992). Note also that the equivalence between the two above-described approaches remains to be proved.

In any case, both model unilateral contact without friction, that is, formally, the following set of boundary conditions on the lips of the crack $\Gamma$ : 


$$
\sigma \cdot v=s v, \quad s \leqslant 0, \quad[u] \cdot v \geqslant 0, \quad s \text { or }[u] \cdot v=0,
$$

in lieu of the no-contact condition

$$
\sigma \cdot v=0
$$

All results of Subsection 4.1 apply to the case of unilateral contact. As alluded to there, the set of bulk-free states could however be empty. In such a case Proposition 4.2 has to be replaced by

Proposition 5.1. If $\mathscr{F}(U)=\varnothing$, then

$$
t_{i}=\infty \text { iff } E_{d}\left(\Gamma, U_{0}\right)=E_{d}\left(\Gamma_{0}, U_{0}\right), \quad \text { for all } \Gamma \supset \Gamma_{0} .
$$

Proof. If $t_{i}=\infty$, then $\Gamma(t)=\Gamma_{0}, t \geqslant 0$. According to (ii) in Law 2.9.

$$
t^{2}\left(E_{d}\left(\Gamma_{0}, U_{0}\right)-E_{d}\left(\Gamma, U_{0}\right)\right) \leqslant E_{s}(\Gamma)-E_{s}\left(\Gamma_{0}\right), \quad \Gamma \supset \Gamma_{0} .
$$

But $E_{d}\left(\Gamma, U_{0}\right) \leqslant E_{d}\left(\Gamma_{0}, U_{0}\right)$, thus, the above relation cannot hold for all $t$ 's unless equality holds. Conversely, if the equality holds, then (iii) of Law 2.9 implies that $E_{s}(\Gamma(t)) \leqslant E_{s}\left(\Gamma_{0}\right)$, which yields the converse.

In other words, no further cracking will occur without a softening of the material. Such is the case of, e.g., compressive 1-D problems without interpenetration.

\subsection{Limits of the current formulation}

As mentioned several times before, our formulation is severely impaired when surface or body force-loads are applied to the sample. Let us briefly explain why it is so. Assume the imposed force-loads are characterized through their work, a linear, bounded functional on $\mathscr{C}(\Gamma, U)$ that we denote by $\mathscr{L}(u)$. It is somewhat natural to replace the bulk energy $E_{d}(\Gamma, U)$ in Laws 2.8 or 2.9 by the potential energy

$$
P(\Gamma, U)=\inf _{v \in \mathscr{C}(\Gamma, U)}\left\{\int_{\Omega_{\Gamma}} W(x, \varepsilon(v)(x)) \mathrm{d} x-\mathscr{L}(v)\right\} .
$$

The effect is however devastating as illustrated below.

Assume, for example, that the only loadings are force-loads $f$ applied to a part, say, $\partial_{f} \Omega$ of the boundary of $\Omega$. Then introduce a crack $\Gamma$ which cuts the domain into two parts $\Omega_{1}$ and $\Omega_{2}$, the boundary of $\Omega_{1}$ containing $\partial_{f} \Omega$. Then a displacement $v$ of the form

$$
v= \begin{cases}s V & \text { on } \Omega_{1}, \\ 0 & \text { on } \Omega_{2},\end{cases}
$$

where $V$ is a constant displacement such that $\mathscr{L}(v)=s \int_{\Omega_{1}} f(x) \mathrm{d} x \cdot V>0$ will be admissible, and its corresponding potential energy will then tend to $-\infty$ as $s$ tends to $\infty$. This would happen for any force-loads such that $\int_{\Omega_{1}} f(x) \mathrm{d} x \neq 0$ if the nocontact condition is adopted, and for appropriately defined tensile force-loads if unilateral conditions prevail. In other words, total failure of the material would occur under any non-zero loading! Not a very encouraging prospect.

Let us suggest several potential remedies. One would view the applied force field as 
$f(x)=\lambda(t) \bar{f}(x)$, where $\bar{f}(x)$ is given, but $\lambda(t)$, the intensity of the force-loads is not. As a balancing factor, we impose an additional kinematic condition, namely that the work of the loads be given, i.e.,

$$
\mathscr{L}(v)=g(t),
$$

where $g$ is some given function of $t$. The break-up of the sample described above cannot occur in such a setting because we would immediately conclude that $\lambda(t)=0$.

A different approach would investigate local minimizers of $P(\Gamma, U)+E_{s}(\Gamma)$. This is unfortunately not feasible as of yet, as emphasized in Subsection 2.2. It might also do away with one of the assets of our formulation, the possibility of brutal crack growth.

\section{REMARKS ON THE MATHEMATICS AND NUMERICS OF THE MODEL}

The proposed formulation could be doomed, its virtues notwithstanding, if not tractable as a well-posed mathematical problem. Let us emphasize right now that the current state of mathematical consistency for the model is at best partial. We give below a few pointers and references for the mathematically inclined reader.

Our minimization principle - especially in its discrete form ; cf Law 2.8 - is strongly reminiscent of the model proposed, in the totally unrelated context of image segmentation, by Mumford and Shah (1989). In that context, they proposed image segmentation through the following algorithm: find a pair $K$, compact of $\Omega$ (the picture) representing the contours of the image in the picture, and $u$, the true pixel intensity at each point of the picture, an element of $\mathscr{C}^{1}(\Omega \backslash K)$, which minimizes

$$
\int_{\Omega \mid \mathrm{K}}|D u|^{2} \mathrm{~d} x+k \mathscr{H}^{N-1}(K)+\int_{\Omega}|u-g|^{2} \mathrm{~d} x,
$$

where $g$ is the measured pixel intensity. It was then shown in De Giorgi et al. (1989) to be equivalent to a well-posed one-field minimization problem on a subspace $S B V(\Omega)$ of the space of functions with bounded variations on $\Omega$, namely,

$$
\int_{\Omega}|\nabla u|^{2} \mathrm{~d} x+k \mathscr{H}^{N-1}(S(u))+\int_{\Omega}|u-g|^{2} \mathrm{~d} x,
$$

where $\nabla u$ represents the absolutely continuous part of the weak derivative of $u$ (a measure), and $S(u)$ the set of jump points for $u$. This was later extended to a problem with Dirichlet boundary conditions on $\partial \Omega$ in Carriero and Leaci (1990).

The analogue result in our setting is not known, except in very specific cases: the antiplane case (Carriero and Leaci, 1990) and the case of an energy density essentially of the form $|\xi|^{p}+g(\xi)$, with $g(\xi) /|\xi|^{p} \rightarrow 0, \xi \rightarrow \infty$ (Fonseca and Fusco, to appear). The obstacles are multiple: firstly, the problem at hand is vector-valued (except in the 1-D or antiplane case); secondly the energy are not quadratic in the field $u$, except in the linearized setting, but, then, symmetrized gradients replace gradients, $S B V$ is not anymore an appropriate space, and $S B D$-type spaces have to be introduced. The structure of $S B D$ spaces is currently the object of active research, see Ambrosio et al. 
(to appear), but, to our knowledge, no results even remotely approaching the equivalence result of De Giorgi et al. (1989) are available. Of course one is always at liberty to substitute the $S B V(S B D)$ setting, in lieu of the setting proposed in this study; that was the approach suggested by Fonseca and Francfort (1995). It is not clear however that this is the proper thing to do. In any case, such an approach will be hard-pressed to evidence the parts of the crack, the lips of which are in contact with one another, an unlikely occurrence in the presence of MIL's, but a more likely one during general loading processes.

A numerical approach based on the $S B V(S B D)$ formulation is currently being developed by Bourdin (to appear) for a description of the approach in the image segmentation setting. It also relies on remarkable $\Gamma$-convergence type approximation results for the minimization of (20) obtained by Ambrosio and Tortorelli (1990). As a side remark, the several approximations proposed by Ambrosio and Tortorelli (1990) look very much like various kinds of damage model, the difference being that they are only used as mathematically convenient approximations of the original discontinuous model, and not as suitable replacements for that model. The numerics are tricky and raise the additional issue of the convergence of the adopted numerical scheme; see Belletini and Coscia (1994) for partial answers in the scalar-valued setting.

Even when the knots are unraveled in the vector-valued and/or symmetrized gradient case, the evolution problem, and most notably item (iii) in Law 2.9, will be yet another hurdle in the investigation of the continuous model. Note that the discrete model would then pose no particular problem; see Fonseca and Francfort (1995).

In conclusion, the mathematical and numerical study of the proposed model is largely untapped, although the approach that uses special spaces of bounded variations seems promising for the time being.

\section{REFERENCES}

Ambrosio, L. (1989) Variational problems in SBV and image segmentation. Acta Appl. Math. 17, 1-40.

Ambrosio, L., Coscia, A. and Dal Maso, G. (to appear) Fine properties of functions with bounded deformations.

Ambrosio, L. and Tortorelli, V. M. (1990) Approximation of functionals depending on jumps by elliptic functionals via $\Gamma$-convergence. Comm. Pure Appl. Math. 43, 999-1036.

Amestoy, M. (1987) Propagations de fissures en élasticité plane. Thèse d'Etat, Paris.

Amestoy, M. and Leblond, J.-B. (1989) Crack path in plane situation-II, detailed form of the expansion of the stress intensity factors. International Journal of Solids and Structures 29(4), 465-501.

Ball, J. M. and James, R. D. (1987) Fine phase mixtures as minimizers of energy. Archives for Rational Mechanics and Analysis 110(1), 13-52.

Barenblatt, G. I. (1962) The mathematical theory of equilibrium cracks in brittle fracture. Advances in Applied Mechanics 7, 55-129.

Belletini, G. and Coscia, A. (1994) Discrete approximation of a free discontinuity problem. Num. Funct. Anal. Optim. 15, 201-224.

Bourdin, B. (to appear in $\mathrm{M}^{2} \mathrm{AN}$ ) Image segmentation with a finite element method.

Carriero, M. and Leaci, A. (1990) Existence theorem for a Dirichlet problem with free discontinuity set. Nonlinear Anal. Th. Meth. Appls. 15(7), 661-667. 
De Giorgi, E., Carriero, M. and Leaci, A. (1989) Existence theorem for a minimum problem with free discontinuity set. Archives for Rational Mechanics and Analysis 108, 195-218.

Ehrlacher, A. and Fedelich, B. (1989) Stability and bifurcation of simple dissipative systems; application to brutal damage. In Cracking and Damage: Strain Localization and Size Effect, ed. J. Mazars and Z. P. Bazant, pp. 217-227. Elsevier, New York.

Evans, L. C. and Gariepy, R. F. (1992) Measure Theory and Fine Properties of Functions. CRC Press, Boca Raton.

Fonseca, I. and Francfort, G. A. (1995) Relaxation in BV versus quasiconvexification in $W^{1, p}$; a model for the interaction between fracture and damage. Calculus of Variations 3(4), 407446.

Fonseca, I. and Fusco, N. (to appear) Regularity results for anisotropic image segmentation models.

Francfort, G. A. and Marigo, J. J. (1993) Stable damage evolution in a brittle continuous medium. Eur. J. Mech. A/Solids 12(2), 149-189.

Francfort, G. A. and Marigo, J. J. (to appear) Cracks in fracture mechanics : a time-indexed family of energy minimizers. In Proceedings of the International IUTAM Symposium, April 1997, Paris: Variations de domaines et frontières libres en mécanique des solides, ed. M. Fremond and Q. S. Nguyen.

Griffith, A. (1920) The phenomena of rupture and flow in solids. Phil. Trans. Roy. Soc. London CCXXI-A, 163-198.

Hashin, Z. (1996) Finite thermoelastic fracture criterion with application to laminate cracking analysis. Journal of the Mechanics and Physics of Solids 44(7), 1129-1145.

James, R. and Kinderlehrer, D. (1993) Theory of magnetostriction with application to $\mathrm{Tb}_{x} \mathrm{D} y_{1-x} \mathrm{Fe}_{2}$. Philosophical Magazine B68, 237-274.

Leblond, J.-B. (1989) Crack paths in plane situation-I, general form of the expansion of the stress intensity factors. International Journal of Solids and Structures 25, 1311-1325.

Leguillon, D. (1990a) Calcul du taux de restitution de l'énergie au voisinage d'une singularité. C.R. Acad. Sci. Paris Série II 309, 945-950.

Leguillon, D. (1990b) Comportement asymptotique du taux de restitution de l'energie en fin de fracture. C.R. Acad. Sci. Paris Série II 310, 155-160.

Mumford, D. and Shah, J. (1989) Optimal approximations by piecewise smooth functions and associated variational problems. Comm. Pure Applied Math. 42, 577-685.

Nguyen, Q. S. (1987) Bifurcation and post-bifurcation analysis in plasticity and brittle fracture. Journal of Mechanics and Physics of Solids 35, 303-324.

Sih, G. C. and Liebowitz, H. (1968) Mathematical theories of brittle fracture. In Fracture: An Advanced Treatise, Vol. II, Mathematical Fundamentals, ed. H. Liebowitz, pp. 67-190. Academic Press, New York. 